\title{
Testicular injury during SARS-CoV-2 infection may be neglected: An assessment from scRNA-seq profiling and protein detection of angiotensin-converting enzyme II
}

\author{
TONGHUI CAI ${ }^{1 *}$, GUANQUAN MAO ${ }^{2 *}$, RUI ZHENG ${ }^{3 *}$, MAO FANG $^{4}$, \\ XIAORONG YANG ${ }^{5}$, LIJING WANG ${ }^{2}$ and CUILING $\mathrm{QI}^{2,6}$
}

\begin{abstract}
${ }^{1}$ Department of Pathology, The Third Affiliated Hospital of Guangzhou Medical University, Guangzhou, Guangdong 510000;
${ }^{2}$ Institute of Basic Medical Sciences, School of Life Sciences and Biopharmaceutics, Guangdong Pharmaceutical University, Guangzhou, Guangdong 510006; ${ }^{3}$ Department of Otolaryngology-Head and Neck Surgery,

The Third Affiliated Hospital, Sun Yat-Sen University, Guangzhou, Guangdong 510630;

${ }^{4}$ Department of Pathology, School of Basic Medical Science, Guangzhou Medical University, Guangzhou, Guangdong 511436;

${ }^{5}$ Clinical Laboratory, The First Affiliated Hospital of Guangdong Pharmaceutical University, Guangzhou, Guangdong 510000;

${ }^{6}$ Guangdong Province Key Laboratory for Biotechnology Drug Candidates,

School of Life Sciences and Biopharmaceutics, Guangdong Pharmaceutical University,
\end{abstract}

Guangzhou, Guangdong 510006, P.R. China

Received July 23, 2020; Accepted December 18, 2020

DOI: $10.3892 / \mathrm{etm} .2021 .10920$

\begin{abstract}
Coronavirus disease 2019 (COVID-19), caused by severe acute respiratory syndrome coronavirus-2 (SARS-CoV-2), is outbreaking globally. SARS-CoV-2 invades host cells via angiotensin-converting enzyme II (ACE2) and causes multiple-organ injury. Autopsy studies indicated that the testis of patients with COVID-19 exhibited various degrees of spermatogenic cell reduction and injury, but the composition of ACE2-expressing cells and their proportion in the testes have remained to be determined. Recent clinical evidence suggested that the ratio of male sex hormones in males with COVID-19 was significantly changed. The present study aimed to explore whether SARS-CoV-2 is able to damage the male reproductive system. For this, the ACE2-expressing cell composition and proportion in male testes were analyzed using single-cell RNA sequencing (RNA-seq) datasets downloaded from the Gene Expression Omnibus (GEO) database and immunohistochemical (IHC) staining. The single-cell
\end{abstract}

Correspondence to: Dr Cuiling Qi or Professor Lijing Wang, Institute of Basic Medical Sciences, School of Life Sciences and Biopharmaceutics, Guangdong Pharmaceutical University, 181 Waihuan East Road, Guangzhou Higher Education Mega Center Panyu, Guangzhou, Guangdong 510006, P.R. China

E-mail: qicuiling12345@163.com

E-mail: wanglijing62@163.com

*Contributed equally

Key words: severe acute respiratory syndrome coronavirus-2, angiotensin-converting enzyme II, orchitis, testis, COVID-19
RNA-seq data indicated that ACE2 mRNA was highly expressed in myoid cells, Leydig cells and spermatogenic cells, accounting for $5.45,1.24$ and $0.423 \%$ of adult testicular cells. ACE2 mRNA-expressing Sertoli cells, spermatogenic cells and myoid cells accounted for 5.00, 0.56 and $0.73 \%$ of infant testicular cells. IHC demonstrated that ACE2 protein was also highly expressed in testicular tissues. In conclusion, the present results demonstrated that testicular injury may be missed by clinicians in patients with COVID-19 and male reproductive function should be closely followed up.

\section{Introduction}

In December 2019, a cluster of patients with pneumonia caused by an unknown pathogen occurred in Wuhan (China) and rapidly spread across the whole country $(1,2)$. Although China took an emergency response in the early days of the outbreak, the infection has been spreading globally. From airway epithelial cell samples, a novel coronavirus was determined by the Chinese Center for Disease Control and Prevention using next-generation sequencing technology and was named severe acute respiratory syndrome coronavirus-2 (SARS-CoV-2) by the World Health Organization. The sequence of SARS-CoV-2 is highly homologous to severe acute respiratory syndrome coronaviruses (SARS-CoV) (3).

Since the sequence of SARS-CoV-2 is highly homologous to that of SARS-CoV, the clinical manifestations of the patients with coronavirus disease 2019 (COVID-19) caused by SARS-CoV-2 are similar to those of patients with SARS and Middle East respiratory syndrome coronavirus (MERS-CoV) (4). The main manifestations are high fever and cough, which may be accompanied by headache, myalgia, joint pain, fatigue, diarrhea and chest tightness at onset. The 
patients may have dyspnea, shortness of breath and then respiratory failure at the later stage $(5,6)$. SARS is a systemic organ damage disease, the main target organs of which are the lung, immune organs and systemic venules. In addition, virus-induced orchitis-like pathological changes were detected during autopsy of numerous patients who succumbed to SARS, even those with no related symptoms during their lifetime (7). Ma et al (8) reported that the ratio of testosterone ( $\mathrm{T}$ ) to serum luteinizing hormone $(\mathrm{LH})$ and the ratio of follicle-stimulating hormone to LH were significantly reduced in patients with COVID-19. Given that the sequences of SARS-CoV-2 and SARS-CoV are highly homologous, SARS-CoV-2 may be likely to injure testicular tissues.

In 2003, angiotensin-converting enzyme II (ACE2) was identified as the functional receptor for SARS-CoV (9), which is able to bind to the receptor-binding domain of viral Spike protein, causing endocytosis and infection (10). It has been reported that SARS-CoV-2 shares the same receptor with SARS-CoV, i.e. ACE2 (11-13). As the majority of ACE2-expressing cells in the respiratory tract are type II alveolar epithelial cells (AT2), SARS-CoV-2 invasion and the corresponding manifestations are mainly caused by interaction with ACE2 receptors on the surface of AT2 (14). A study has indicated that ACE2-expressing epithelial cells of the small intestine were susceptible to SARS-CoV-2 infection (15).

SARS-CoV-2 has been reported to damage multiple organs and alter the ratio of male sex hormones in males with COVID-19 (8); however, its direct effect on the testis has remained to be fully determined. In the present study, the expression profiles of ACE2 were investigated by analyzing single-cell RNA-sequencing (RNA-seq) datasets and immunohistochemical (IHC) staining to explore the expression of ACE2 and assess the effect of SARS-CoV-2 on the male productive function.

\section{Materials and methods}

Data sources. Single-cell RNA-seq datasets (nos. GSE120506 and GSE112013) were downloaded from the Gene Expression Omnibus (GEO) database (https://www.ncbi.nlm.nih. gov/geo/). RNA-seq data of normal human tissues from the GTEx database were downloaded using the University of California Santa Cruz (UCSC) Xena browser (https://xena. ucsc.edu/).

ACE2 expression patterns in different tissues from normal human subjects from GTEx. The dataset gene expression RNAseq-TOIL RSEM fragments per kilobase of exon model per million mapped fragments and dataset phenotype-GTEx phenotype were downloaded from UCSC Xena. Strawberry-perl 5.3.2.1 (https://strawberryperl.com/) was used to quantify the mRNA expression of ACE2 in each organ. The R package 'ggpubr' was used to draw boxplots of ACE2 mRNA expression in each organ.

Single-cell sequencing analysis. Single-cell RNA-seq datasets (nos. GSE120506 and GSE112013) were downloaded from the GEO database. The Single cell RNA sequencing (scRNA-seq) datasets of GSE112013_Combined_UMI_table.txt and GSE120506_infant_combined_UMI.txt were subsequently analyzed using the R package Seurat (version 3.1.2). Strict parameters, ' $\mathrm{min}$.cells $=3$ ' and ' $\mathrm{min}$. features $=50$ ', were used to filter out low-quality cells and low-quality genes in the function CreateSeuratObject. Subsequently, the data were log-normalized using the function NormalizeData with the default parameters. The genes, with the largest standard deviation of expression in all cells were determined by the function FindVariableGenes. After the data were dealt with, the function ScaleData, principal component analysis (PCA) dimensionality reduction was performed by using the function RunPCA. By using the functions of JackStraw and ScoreJackStraw, the PCA components with $\mathrm{P}<0.05$ were selected for further analysis using two-dimensional t-distributed stochastic neighbor embedding. The setting of k.param was 20 in the function FindNeighbors and the setting of the resolution was 0.5 in the function FindClusters. The cell types were determined according to the expression of marker genes. Gene expression of different cell types was displayed using the functions DotPlot and VlnPlot.

IHC staining of human samples. Human normal testicular tissue sections $(n=4)$ included in normal tissue microarrays were obtained from Alenabio. In addition, the human testicular tissues from 11 patients with prostate cancer were obtained from the Third Affiliated Hospital of Guangzhou Medical University (Guangzhou, China). The specimen collection started on 1 September 2020 and was completed by 31 October 2020. For IHC staining, 3- $\mu \mathrm{m}$ sections were prepared. IHC staining was performed as described in a previous study by our group (16). In brief, the sections were dewaxed, hydrated and incubated with anti-ACE2 antibody (1:400 dilution; cat. no. ab15348; Abcam) overnight at $4^{\circ} \mathrm{C}$. Subsequently, HRP-conjugated secondary antibody (1:100 dilution; cat. no. BM3894; Boster Biotechnology, Inc.) was added to the sections and incubated for $50 \mathrm{~min}$ at $37^{\circ} \mathrm{C}$. All of the sections were stained with diaminobenzidine and counterstained with hematoxylin. Images were captured using an upright microscope and the expression levels of ACE2 were evaluated by two experimenters. The data were independently collected and evaluated by two experimenters (GM and RZ) in a double-blind manner.

Statistical analysis. The Graphpad Prism 5 software package (GraphPad Software, Inc.) was used to analyze the data and draw the statistical charts. The two-tailed Student's t-test was used to statistically analyze the results. $\mathrm{P}<0.05$ was considered to indicate a statistically significant difference.

\section{Results}

ACE2 mRNA expression in different organs of normal human subjects. ACE2 mRNA expression was detected in different organs of normal human subjects by analyzing RNA-seq data from the GTEx database. ACE2 mRNA was indicated to be highly expressed both in the small intestine and testis (Fig. 1). These results indicated that testis may be susceptible to SARS-CoV-2 because SARS-CoV-2 invades host cells via ACE2 receptors.

Identification of cell types in the testes of adults and infants. Given that SARS-CoV-2 invades host cells via ACE2 receptors 


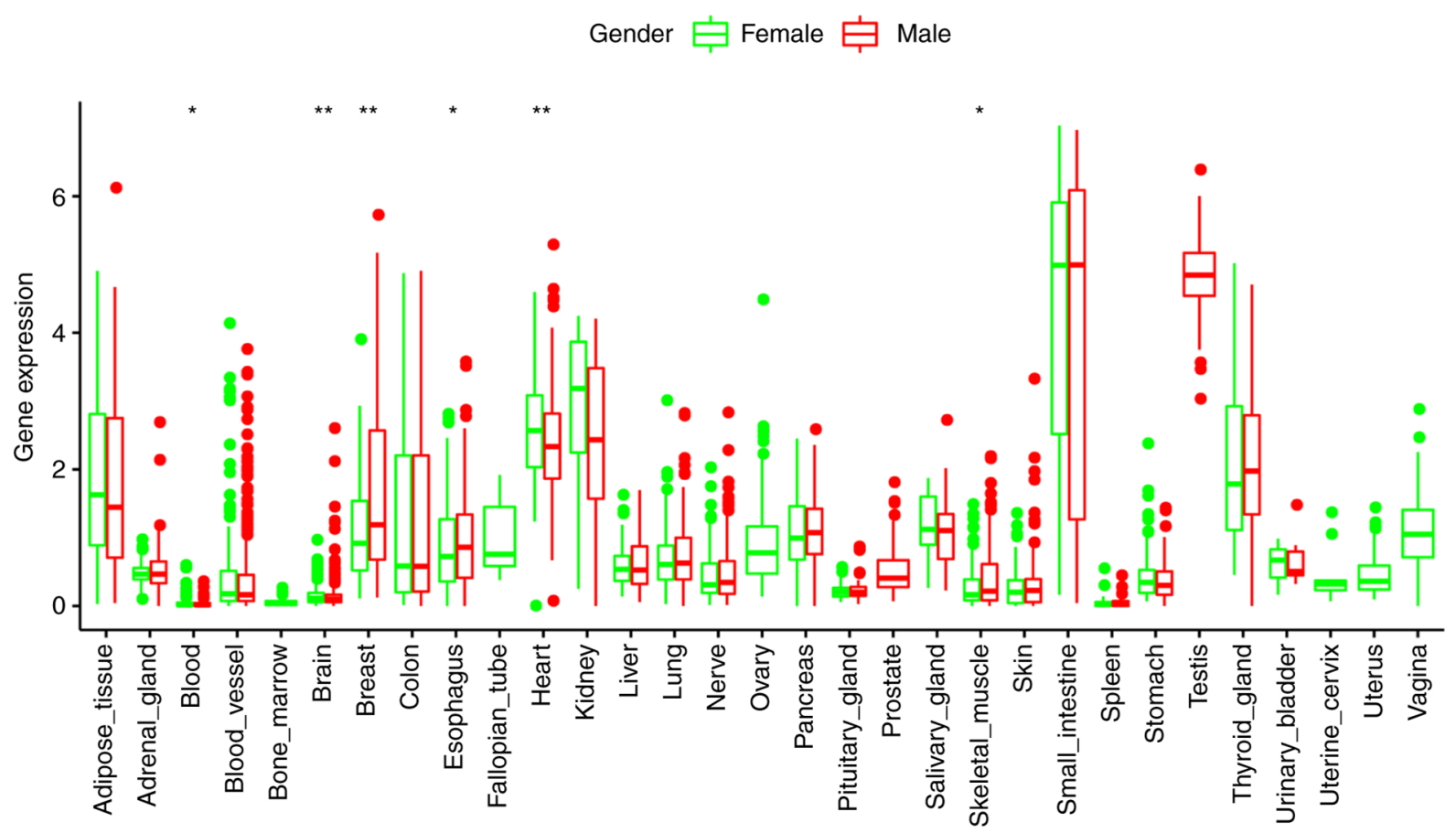

Figure 1. ACE2 mRNA expression in different organs of normal human subjects according to the GTEx database. ACE2 mRNA was highly expressed in normal testis $(\mathrm{TPM}(\log 2)=5)$. The box-plot presented the expression of ACE2 mRNA in various organs. The middle line of the box-plot represents the median value. The higher the median, the higher the gene expression. ${ }^{\mathrm{P}}<0.05 ;{ }^{* *} \mathrm{P}<0.01$, female vs. male. ACE2, angiotensin-converting enzyme II.

and SARS-CoV is able to cause orchitis (7), ACE2-expression by cell type was detected in the present study by analyzing single-cell RNA-seq data. The R package Seurat was used to re-analyze the scRNA-seq datasets GSE112013 and GSE120506. In order to normalize the data, the data of the datasets were filtered and normalized. After the data were normalized, 1,500 genes from GSE112013 (Fig. S1) and 5,000 genes from GSE120506 (Fig. S2), with larger coefficients of variation ( $\min$ cell $=3$, min features $=50$ ), were selected for subsequent analysis and the top 10 genes with the largest standard deviation were displayed. In order to reduce the linear dimensionality, a PCA algorithm was used to analyze the dataset. It was indicated that the PCs $(\mathrm{P}<0.05)$ of the dataset from the adult testes (Fig. S3) and the infant testes (Fig. S4) were suitable for further analysis.

A total of 3,652 individual cells from the testes of three healthy males of peak reproductive age and 813 individual cells from the testes of two infants (age, 13 months) were analyzed. Using unsupervised graph-based clustering, it was indicated that at least 12 distinct cell clusters were present in the testicular tissues of the adult males (Fig. 2A and B) and six cell clusters were identified in the infant testicular tissues (Fig. 2C and D) according to their corresponding marker gene expression profiles.

Identification of ACE2-expressing cells in adult testes. To determine the cell types expressing ACE2, the mRNA expression of ACE2 was analyzed from scRNA-seq datasets. The violin plots indicated that ACE2 was highly expressed in spermatogenic cells, Leydig cells and myoid cells of the adult male testicular tissues, but hardly expressed in endothelial cells and macrophages (Fig. 3A). Of note, investigation of the expression profiles of another virus receptor, dipeptidyl peptidase 4 (DPP4) receptor for MERS-CoV virus, indicated that DPP4 was highly expressed in spermatogenic cells, Leydig cells and endothelial cells of the adult male testicular tissues, while its expression was low in myoid cells and macrophages. Furthermore, the expression of DPP4 in spermatogenic cells was higher compared with that of ACE2 (Fig. 3B). ACE2-expressing myoid cells, Leydig cells and spermatogenic cells were determined to account for 5.45, 1.24 and $0.423 \%$ of adult testicular cells, respectively (Fig. 3C). Furthermore, DPP4-expressing spermatogenic cells, Leydig cells and endothelial cells accounted for 6.91, 2.48 and $1.56 \%$ of adult testicular cells, respectively (Fig. 3D). These results indicated that SARS-CoV-2 and MERS-CoV may damage the adult testis, as these two viral receptors have significantly overlapped and increased expression profiles.

Identification of ACE2-expressing cells in infant testes. To predict the effect of SARS-CoV-2 on infant testes and determine the cell types expressing ACE2 in infant testes, the mRNA expression of ACE2 in infant testicular tissues was also analyzed. It was determined that ACE2 was highly expressed in Sertoli cells of the infant testicular tissues, but hardly expressed in spermatogenic cells and myoid cells (Fig. 4A). ACE2-expressing Sertoli cells, spermatogenic cells and myoid cells accounted for 5.00, 0.56 and $0.73 \%$ of infant testicular cells, respectively (Fig. 4B). These results suggested that SARS-CoV-2 may also damage the testes of infants.

ACE2 protein is highly expressed in normal testicular tissues. To determine the expression of ACE2 protein in normal testicular tissues, the testicular tissues from 15 patients with prostate cancer were sectioned and subjected to IHC analysis. IHC staining for ACE2 was performed on the testicular 
A

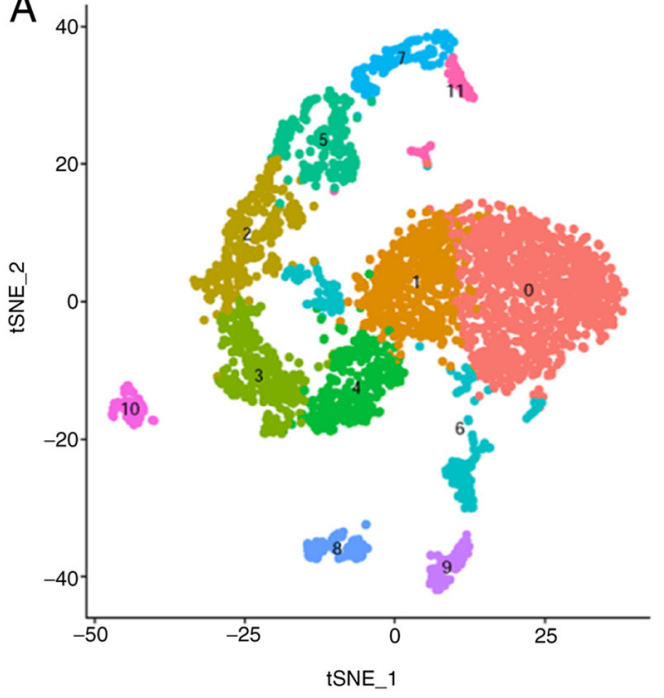

$\bullet 0 \bullet 1 \bullet 2 \bullet 3 \bullet 4 \bullet 5 \bullet 7 \bullet 11$ spermatogenic cells $\bullet 6$ Leydig cells - 8 endothelial cells 9 myoid cells 10 macrophages

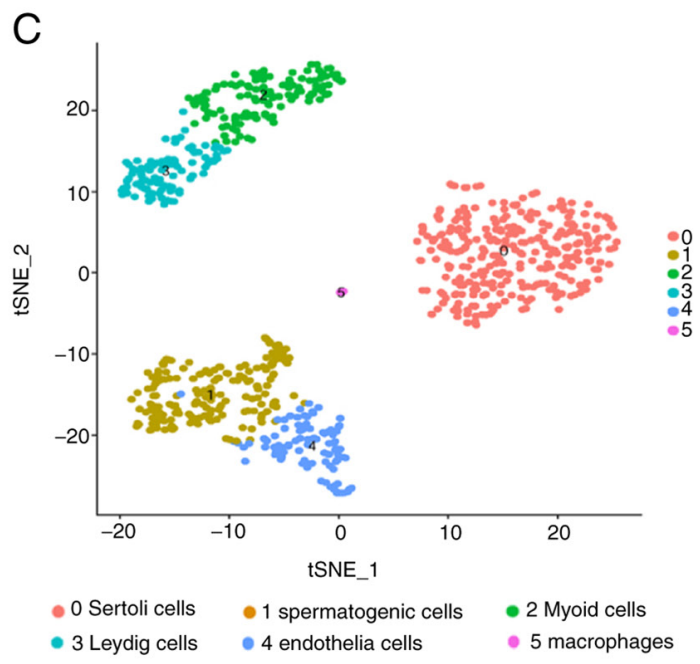

B

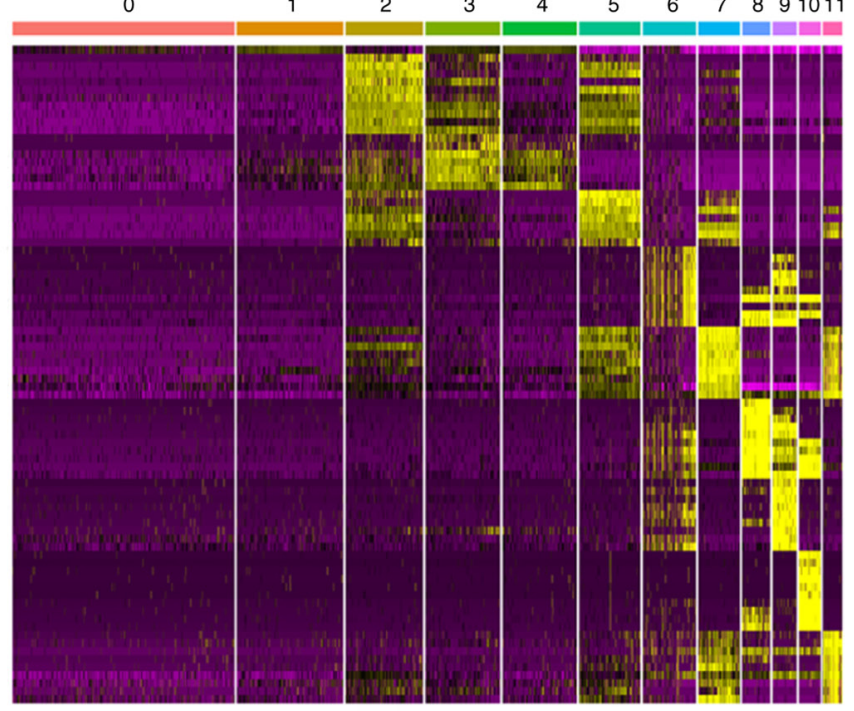

D

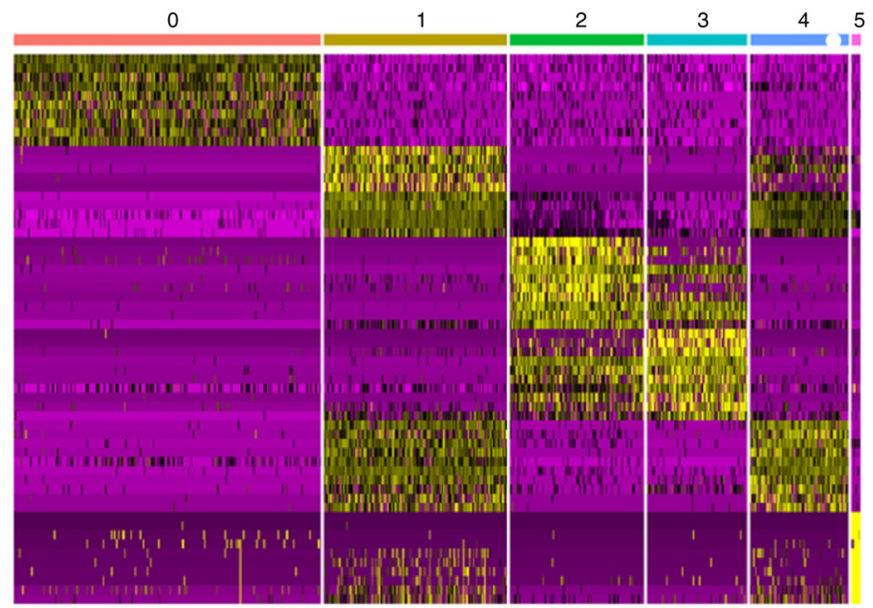

Figure 2. Single-cell atlas of adult and infant testes. (A) Scatter and (B) heatmap analysis of single-cell sequencing data identified twelve cell sub-clusters in adult testicular cells. (C) Scatter and (D) heatmap analysis of single-cell sequencing data identified six cell sub-clusters in infant testicular cells; tSNE, two-dimensional t-distributed stochastic neighbor embedding.

tissue sections. The results indicated that ACE2 protein was highly expressed in the normal testicular tissues, including spermatogenic cells, Sertoli cells, interstitial cells and myoid cells (Fig. 5A and B). These results further demonstrated that the testis may be target organs of SARS-CoV-2 and imply that SARS-CoV-2 may cause testicular damage and impair reproductive function, and even lead to male infertility.

\section{Discussion}

Recently, COVID-19 has caused a great health threat throughout the world and led to the declaration of a public health emergency of international concern by the World Health Organization $(6,13)$. The pathogen is SARS-CoV-2, a single-stranded RNA virus, which has a stronger transmission ability than SARS. Apart from lung injury as the most common manifestation, COVID-19 is able to cause systemic/multiple-organ damage, including the heart, kidney and liver. However, whether SARS-CoV-2 infects the testis and causes damage to male fertility function has remained elusive.

As mentioned above, as ACE2 is the cell entry receptor for SARS-CoV-2, it is worthwhile to detect ACE2 expression in testicular tissue in order to explore whether male testes are a potential target of virus invasion. In the present study, single-cell RNA-seq expression profiles were analyzed to reveal that ACE2 was highly expressed in spermatogenic cells, Leydig cells and myoid cells of adult testes and Sertoli cells of infant testicular tissues, which demonstrated that not only lung and heart but also testes are potential target organs. These results suggest that SARS-CoV-2 may cause testicular damage and impair reproductive function, even leading to male infertility.

By investigating the expression of ACE2 in normal human testicles, it was revealed that ACE2 protein was highly expressed in spermatogenic cells, Leydig cells and myoid cells of the adult testes. Ding et al (17) reported that SARS-CoV was detected in numerous organs and tissues, including the lung, trachea, bronchus, stomach and small intestine, by using 

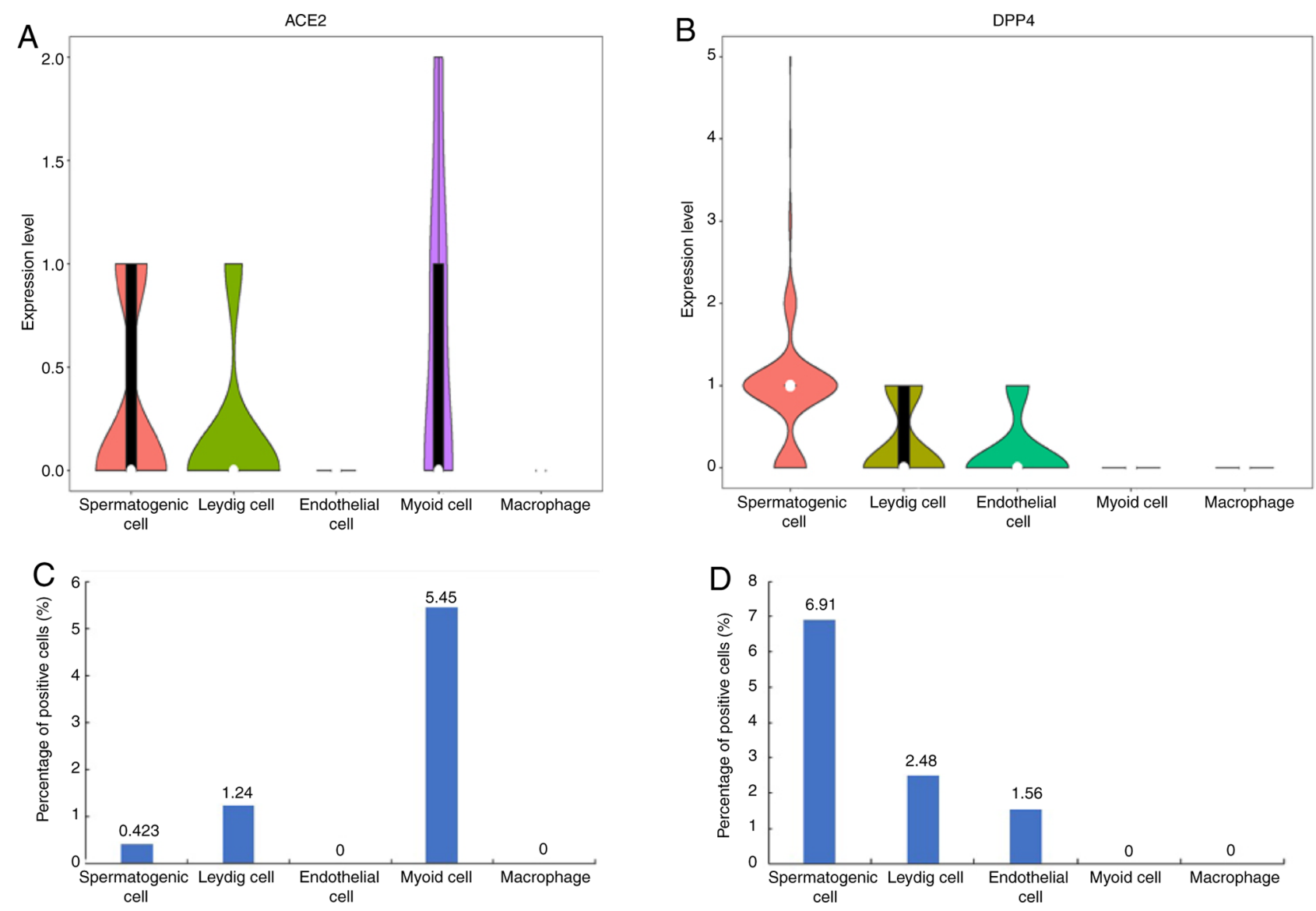

Figure 3. Expression profiles of ACE2 and DPP4 in adult testicular cells. (A) Violin plot of the expression profiles of SARS-CoV and SARS-CoV-2 receptor ACE2 in adult testicular cells. The black rectangle represents the range from the lower quartile to the upper quartile. The upper edge of the rectangle represents the upper quartile. The lower edge represents the lower quartile. ACE2 was highly expressed in myoid cells, Leydig cells and spermatogenic cells. (B) Violin plot for the expression profile of middle East respiratory syndrome-CoV receptor DPP4. DPP4 was highly expressed in spermatogenic cells, Leydig cells and endothelial cells. (C) Percentage of ACE2-expressing cells in adult testicular cells. (D) Percentage of DPP4-expressing cells in adult testicular cells. ACE2, angiotensin-converting enzyme II; DPP4, dipeptidyl peptidase 4; SARS-CoV-2, severe acute respiratory syndrome coronavirus-2.
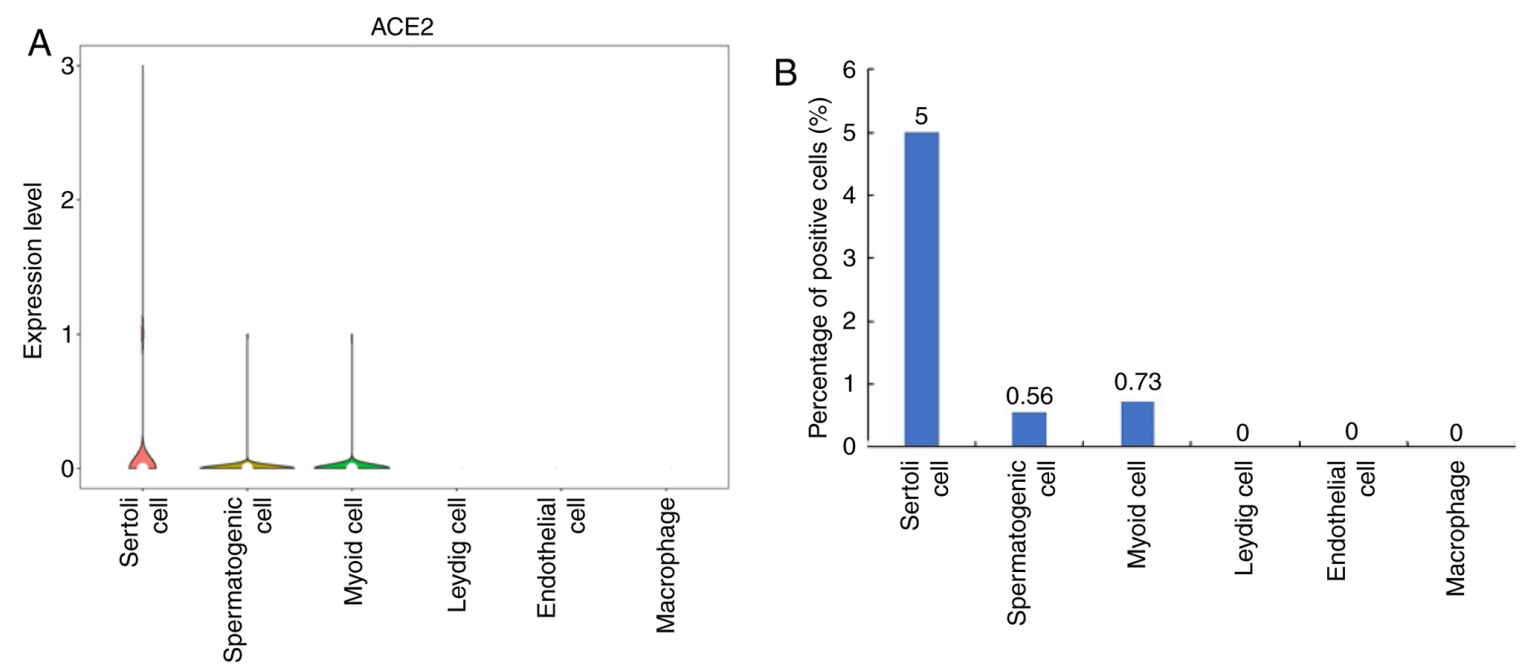

Figure 4. Expression profiles of ACE2 in the infant testicular cells. (A) Violin plot of the expression profiles of SARS-CoV and SARS-CoV-2 receptor ACE2 in infant testicular cells. ACE2 was highly expressed in Sertoli cells. (B) Percentage of ACE2-expressing cells in infant testicular cells. ACE2, angiotensin-converting enzyme II; SARS-CoV-2, severe acute respiratory syndrome coronavirus-2.

IHC and in situ hybridization, but not in the testes. However, Zhao et al (18) detected SARS-CoV in the seminiferous epithelial and mesenchymal cells of the testes using electron microscopic examination of post-mortem materials. In the present study, the expression of ACE-2 in tissues from patients with COVID-19 was not examined, as it was not possible 


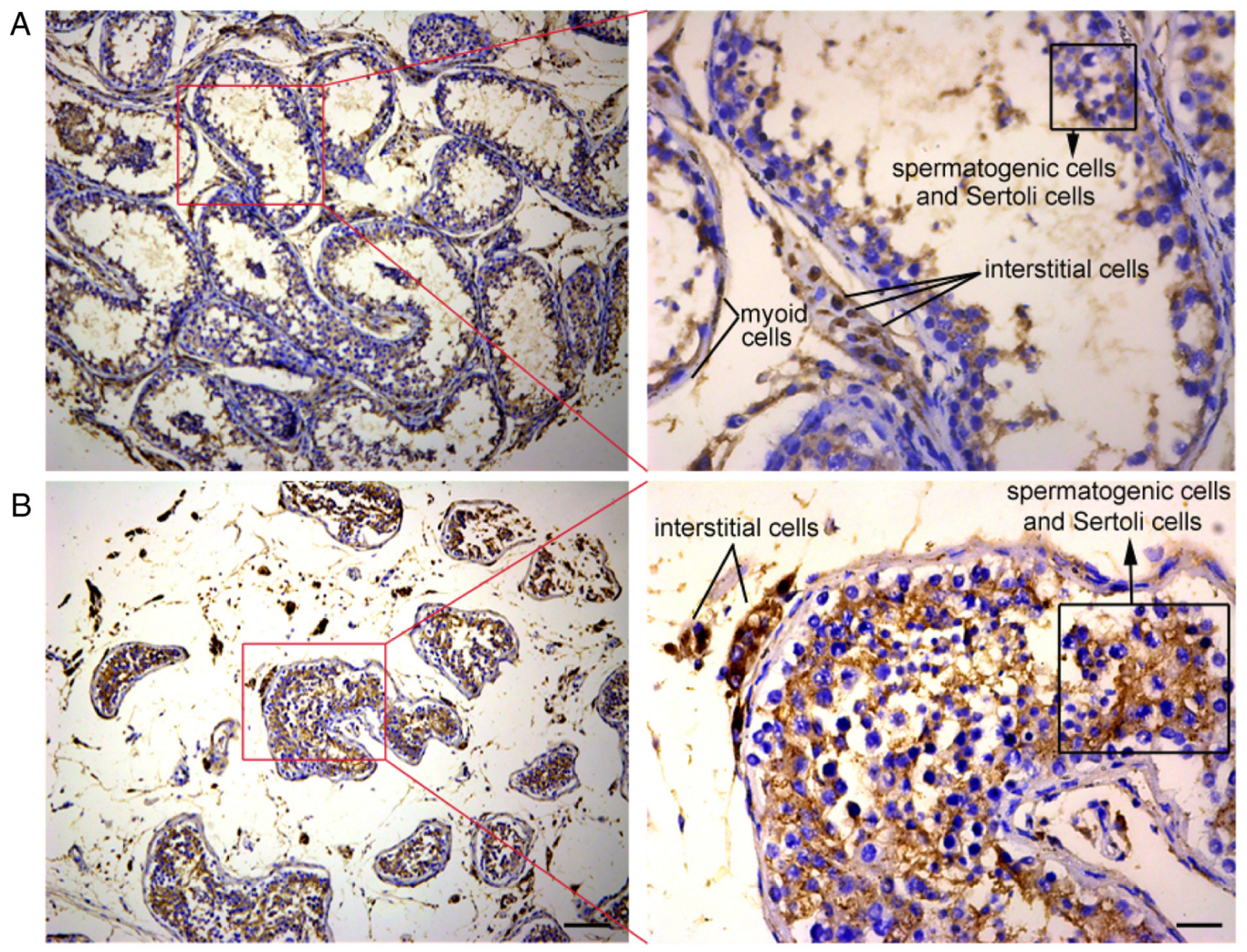

Figure 5. ACE2 protein is highly expressed in normal testicular tissues. IHC staining of ACE2 was used to determine whether ACE2 was expressed in the normal testicular tissues from 15 patients with prostate cancer. IHC images of male testicular tissues from two patients, (A) case 1 and (B) case 2 . The right panels demonstrate the appearance of seminiferous tubules and interstitial cells at higher magnification as indicated by red squares in the left panels. ACE2 was highly expressed in spermatogenic cells, Sertoli cells, interstitial cells and myoid cells (scale bars, $100 \mu \mathrm{m}$ ). ACE2, angiotensin-converting enzyme II; IHC, immunohistochemistry.

to obtain tissues from post-mortem patients. Since ACE2 is highly expressed in testicular cells, it may be speculated that the testis are also a potential target of SARS-CoV-2 infection.

SARS-CoV is able to cause testicular damage, manifested as a thickened basement membrane of seminiferous tubules and fibrosis of seminiferous tubules. The seminiferous epithelial cells were necrotic and fell off to different degrees, resulting in a significant decrease in the number of spermatogenic cells. Extensive germ cell destruction with few or no spermatozoa in the lumen of seminiferous tubules were observed. Vascular congestions, vasodilation and leukocyte infiltration were present in the testicular stroma (7). Furthermore, Ma et al (8) indicated that serum LH and PRL levels were significantly elevated and the ratio of T:LH was significantly decreased in patients with COVID-19, implying dysfunction of the testes. Xu et al (1) also reported that RNA and viral particles of SARS-CoV-2 were detected in testes, and testes were subjected to various degrees of spermatogenic cell reduction and injury. The present results also suggested that ACE2 mRNA and protein were highly expressed in the testis. SARS-CoV-2 invade into the cells via the ACE2 receptor, the same as SARS-CoV. Therefore, SARS-CoV-2 may cause severe pathological damage in the testes of infants and adults.

It has been reported that the virus not only causes orchitis but also causes infertility and even increases the incidence of testicular cancer. 59.2\% of patients with COVID-19 were reported to be male, of whom $56 \%$ were $0-49$ years old. Therefore, close attention should be paid for the reproductive function of male inpatients with COVID-19, particularly those with severe disease. If clinically cured patients were to be further followed up and were subjected to periodic comprehensive testing of the sperm count, morphology and vitality, it would further the understanding of COVID-19, and is directly related to the recovery of patients with COVID-19 and their quality of life.

\section{Acknowledgements}

Not applicable.

\section{Funding}

This work was supported by grants from the National Science Foundation of China (grant no. 81773095 to CQ and 31771578 to LW) and the Youth Research Fund of the Third Affiliated Hospital of Guangzhou Medical University (grant no. 2017Q12 to TC).

\section{Availability of data and materials}

The single scRNA-seq datasets generated and/or analyzed during the current study are available in the GEO repository (https://www.ncbi.nlm.nih.gov/geo/query/acc. cgi?acc=GSE120506 and https://www.ncbi.nlm.nih.gov/geo/ query/acc.cgi?acc=GSE112013).

\section{Authors' contributions}

CQ and LW conceived and designed the study. TC and MF acquired and analyzed the single-cell RNA-seq data. RZ and 
GM performed the IHC staining. TC and CQ performed the statistical analysis. XY evaluated the immunohistochemical results, reviewed the manuscript as a clinical laboratory specialist and provided critical guidance in the process of manuscript revision. CQ wrote and modified the manuscript. CQ and TC confirmed the authenticity of the raw data. All authors read and approved the final manuscript.

\section{Ethics approval and consent to participate}

All patients provided written informed consent for their tissues to be used for medical research. Ethical approval for this study was obtained from the Ethics committee of the Third Affiliated Hospital of Guangzhou Medical University (Guangzhou, China).

\section{Patient consent for publication}

Not applicable.

\section{Competing interests}

The authors declare that they have no competing interests.

\section{References}

1. Xu B, Yao XH, Ping YF, Yu SC, Shi Y, Luo T, et al: Autopsy of COVID-19 victims in China. National Science Review 2020 https://academic.oup. com/nsr/advance-article/doi/10.1093/nsr/ nwaa123/5854209? searchresult $=1$.

2. Chen N, Zhou M, Dong X, Qu J, Gong F, Han Y, Qiu Y, Wang J, Liu Y, Wei Y, et al: Epidemiological and clinical characteristics of 99 cases of 2019 novel coronavirus pneumonia in Wuhan, China: A descriptive study. Lancet 395: 507-513, 2020.

3. Hayashi T, Abiko K, Mandai M, Yaegashi N and Konishi I: Highly conserved binding region of ACE2 as a receptor for SARS-CoV-2 between humans and mammals. Vet Q 40: 243-249, 2020.

4. Zhou J, Li C, Zhao G, Chu H, Wang D, Yan HHN, Poon VKM, Wen L, Wong BHY, Zhao X, et al: Human intestinal tract serves as an alternative infection route for Middle East respiratory syndrome coronavirus. Sci Adv 3: eaao4966, 2017.

5. Guan WJ, Ni ZY, Hu Y, Liang WH, Ou CQ, He JX, Liu L, Shan H, Lei CL, Hui DSC, et al: Clinical characteristics of coronavirus disease 2019 in China. N Engl J Med 382: 1708-1720, 2020.

6. Huang C, Wang Y, Li X, Ren L, Zhao J, Hu Y, Zhang L, Fan G, $\mathrm{Xu}$ J, Gu X, et al: Clinical features of patients infected with 2019 novel coronavirus in Wuhan, China. Lancet 395: 497-506, 2020
7. Xu J, Qi L, Chi X, Yang J, Wei X, Gong E, Peh S and Gu J: Orchitis: A complication of severe acute respiratory syndrome (SARS). Biol Reprod 74: 410-416, 2006.

8. Ma L, Xie W, Li DY, Shi L, Mao Y, Xiong Y, Zhang Y and Zhang M: Effect of SARS-CoV-2 infection upon male gonadal function: A single center-based study. medRxiv: Mar 30, 2020 (Epub ahead of print). doi: org/10.1101/2020.03.21.20037267.

9. Li W, Moore MJ, Vasilieva N, Sui J, Wong SK, Berne MA, Somasundaran M, Sullivan JL, Luzuriaga K, Greenough TC, et al: Angiotensin-converting enzyme 2 is a functional receptor for the SARS coronavirus. Nature 426: 450-454, 2003.

10. Kuiken T, Fouchier RA, Schutten M, Rimmelzwaan GF, van Amerongen G, van Riel D, Laman JD, de Jong $T$, van Doornum G, Lim W, et al: Newly discovered coronavirus as the primary cause of severe acute respiratory syndrome. Lancet 362: 263-270, 2003.

11. Wan Y, Shang J, Graham R, Baric RS and Li F: Receptor recognition by the novel coronavirus from Wuhan: An analysis based on decade-long structural studies of SARS Coronavirus. J Virol 94: e00127, 2020.

12. Letko M, Marzi A and Munster V: Functional assessment of cell entry and receptor usage for SARS-CoV-2 and other lineage B betacoronaviruses. Nat Microbiol 5: 562-569, 2020.

13. Zhou P, Yang XL, Wang XG, Hu B, Zhang L, Zhang W, Si HR, Zhu Y, Li B, Huang CL, et al: A pneumonia outbreak associated with a new coronavirus of probable bat origin. Nature 579: 270-273, 2020 .

14. Zhang H, Kang Z, Gong H, Xu D, Wang J, Li Z, Li Z, Cui X, Xiao J, Zhan J, et al: Digestive system is a potential route of COVID-19: An analysis of single-cell coexpression pattern of key proteins in viral entry process. Gut 69: 1010-1018, 2020.

15. Liang W, Feng Z, Rao S, Xiao C, Xue X, Lin Z, Zhang Q and Qi W: Diarrhoea may be underestimated: A missing link in 2019 novel coronavirus. Gut 69: 1141-1143, 2020.

16. Qi C, Wei B, Zhou W, Yang Y, Li B, Guo S, Li J, Ye J, Li J, Zhang Q, et al: P-selectin-mediated platelet adhesion promotes tumor growth. Oncotarget 6: 6584-6596, 2015.

17. Ding Y, He L, Zhang Q, Huang Z, Che X, Hou J, Wang H, Shen H, Qiu L, Li Z, et al: Organ distribution of severe acute respiratory syndrome (SARS) associated coronavirus (SARS-CoV) in SARS patients: Implications for pathogenesis and virus transmission pathways. J Pathol 203: 622-630, 2004.

18. Zhao JM, Zhou GD, Sun YL, Wang SS, Yang JF, Meng EH, Pan D, Li WS, Zhou XS, Wang YD, et al: Clinical pathology and pathogenesis of severe acute respiratory syndrome. Zhonghua Shi Yan He Lin Chuang Bing Du Xue Za Zhi 17: 217-221, 2003 (In Chinese). 